\title{
DISCORSO SULL'ARTE
}

\author{
Piermario Vescovo (Università Ca’ Foscari, Venezia)
}

Cita Recomendada: Piermario Vescovo, «Discorso sull'Arte», Anuario Lope de Vega. Texto, literatura, cultura, XXIII (2017), pp. 203-228.

DOI: <http://dx.doi.org/10.5565/rev/anuariolopedevega.207>

Fecha de recepción: 3 de mayo de 2016

Fecha de aceptación: 24 de mayo de 2016

\section{RIASSUNTO}

Risalendo all'indietro, da Goldoni a Cervantes e a Lope, il contributo prova ad inquadrare l'eredità lontana dei termini "comedia» e «arte», nella discesa dall'Ars poetica, secondo una linea non normativa, nella fondazione di un'ars drammatica o «comica» nel rapporto tra scrittura e pubblico nell'Europa moderna. Rapporto che è peraltro il centro originale dell'antico discorso di Orazio.

Parole chiave: Goldoni; Cervantes; Lope; Ars comica; Commedia dell'arte.

\section{Abstract}

The essay contextualizes the ancient terms «comedia» and «arte» from Goldoni, Cervantes and Lope to Ars poetica, according to a non-normative line, in the creation of an ars dramatica or "comica" in the relationship between writing and the public in modern Europe, that it is also the original center of Horace's discourse.

KeYwords: Goldoni; Cervantes; Ars comica; Commedia dell'arte.

1. La parte centrale riprende, in forma scritta e ripensata, un intervento fatto nell'ambito dell'VIII Congreso Internacional Lope de Vega, intitolato a Lope y el teatro europeo de su tiempo. Le citazioni dal Don Quijote fanno riferimento all'edizione a cura di Francisco Rico [2004] e per l'Arte nuevo de hacer comedias, all'edizione di Enrique García Santo-Tomás [2006]. Un ringraziamento all'amica Valle Ojeda per la lettura in anteprima dei suoi recenti studi cervantini. 


\section{Da Goldoni a Cervantes}

Nel loro palazzo (antichissima abitazione de' Memi sino ai primi tempi della Repubblica) venni per essere favorito da uno e partii onorato da due. Mi fu concesso in quel giorno che io potessi decorare le opere della mia edizione col nome grande di una sì illustre famiglia e per colmo di grazia che potessi imprimere, fra i nomi de' miei protettori, quelli di due fratelli di tanta virtù e di tanta gentilezza. Viveva in allora l'eccellentissimo signor cavaliere Andrea di gloriosissima ricordanza, stella luminosissima di questo cielo, che nei governi e nel senato e nel collegio serenissimo e dapertutto e sempre, fe' salire sino all'ultimo grado la sua virtù, il suo zelo e l'amore per la patria, per la verità e per la giustizia, nemico dell'interesse, nemico della vanità e della pompa e amico solo del pubblico bene, al quale ha consacrato tutti i giorni della sua vita [...] Ei, che pensava sempre alla felicità del paese, credeva con l'egregio, celebratissimo Muratori e coll'eruditissimo marchese Maffei e con tanto altri antichi e moderni saggi, accreditati scrittori, che le morate commedie utile cosa fossero e da desiderarsi da un ben regolato governo. ${ }^{2}$

Questo passo rappresenta la parte essenziale —in cui cade una definizione memorabile - della dedica dell'Uomo di mondo di Carlo Goldoni ai fratelli Andrea e Bernardo Memmo, patrizi veneti e uomini di Stato. La commedia appare nel tomo X dell'edizione Paperini (1757) e richiama la sottoscrizione della stessa da parte dei dedicatari al suo avvio. La rievocazione della figura del loro zio, Andrea Memmo senior, morto nel 1754, permette al commediografo di retrocedere ancora nel tempo, di ricordare l'interesse da costui manifestato (nonostante «i gravissimi pesi della Repubblica») per le sue «comiche fatiche» e di collocare ancora più indietro il nesso tra (le sue) morate commedie e ben regolato governo. Un Goldoni, dunque, che nella seconda metà degli anni '50 retrodata, in questo senso, la categoria di Riforma, posto che la dedica accompagna addirittura la sua prima commedia (debitamente riscritta) e il suo esordio sulla scena del «Teatro Comico». ${ }^{3}$

2. Carlo Goldoni, Tutte le opere, p. 777.

3. L'etichetta è comunque attestata - al netto delle problematizzazioni da parte della critica sul suo significato - nel riferimento all'azione di Goldoni fin dalla pubblicistica e pamphlettistica dei primi anni cinquanta, come documenta il libretto — uscito per i tipi di Giuseppe Bettinelli nel 1752_- 
La «Repubblica» è qui quella veneziana e la citazione del nome di Muratori (e del suo Della pubblica felicità, il cui sottotitolo suona, del resto, Oggetto de' buoni Principi) amplia il raggio puntualmente politico del riferimento in rapporto alla felicità del paese (accanto il nome del marchese Maffei, che aveva, tra l'altro, scritto un trattato sulla funzione del teatro: figure caratterizzanti di quello che possiamo, con Franco Venturi, definire il Settecento riformatore). ${ }^{4}$

Ma chi sono gli «altri antichi e moderni saggi e accreditati scrittori» cui allude Goldoni? Si tratta di una mera formula di rito? Il rinvio va a una tradizione centonaria sull'utilità e moralità della commedia, contro ai suoi detrattori, o possiede relazioni più precise? Ora, un'affermazione pressoché identica si incontra, non senza sorpresa, nel Don Quijote: un'opera che contiene, tra le innumerevoli altre cose, negli ultimi capitoli della prima parte, anche una celeberrima discussione tra il canonico e il curato sulla dignità e la liceità della comedia, mentre essi accompagnano per via il carretto che riporta a casa, ingabbiato, il cavaliere dalla triste figura.

Solo per rammentare il punto di inserimento del discorso in questione, basterà aggiungere che il riferimento cade all'interno, o a lato, della più ampia discussione dedicata a la materia de los libros de caballerías, vale a dire che il discorso sulla commedia è, precisamente, una parentesi che si apre all'interno o in appendice a quello dedicato al romanzo, alla sua varietà e alla ricchezza dell'eredità - dunque a quello che Cervantes sta costruendo e tramando- dei libri che Don Quijote ha raccolto nella sua biblioteca, già messi molti capitoli prima al rogo dal curato, per provare a guarire la pazzia del proprietario o solo per esorcizzarla. Ma nella condanna qui pronunciata dal canonico, nel suo disprezzo per le assurdità e inverosimiglianze del romanzo cavalleresco, emerge in contropiano una ben diversa attenzione, che ribalta la mescolanza e disomogeneità del genere in ricchezza, in cui sta evidentemente il fondamento dell'impresa cervantina: «Porque la escritura desatada destos libros da lugar a que el autor pueda mostrarse épico, lírico, trágico, cóm-

Della Commedia italiana e delle sue regole ed attinenze (di Giuseppe Antonio Costantini, contro Il marito dissoluto di Francesco Griselini); si veda la battuta d'attacco del Cavaliere allo Studioso all'inizio del dialogo: «Avete voi notato la grande riforma introdotta da tre o quattro anni in qua nel Teatro Comico dell'Italia?» (p. 1). Teatro comico è qui impiegato nell'accezione di «teatro dei comici di professione» (si veda a pp. 2-3, chiarissimo: «Chi opera per mercede, può esser buono, mediocre e infelice; e talora per uniformarsi al genio di chi dà la mercede, o all'inabilità di chi rappresenta, convien che faccia delle mostruosità»). La scrittura per la scena e gli autori che la praticano si inseriscono, dunque, in questa prospettiva.

4. Ho già analizzato questi aspetti nel mio La felicità delle commedie (Vescovo 2016). 
ico, con todas aquellas partes que encierran en si las dulcísimas y agradables ciencias de la poesía y de la oratoria; que la épica también puede escrebirse en prosa como en verso".

Così termina il capitolo quarantasettesimo; si apra, quindi, il seguente, dove si incontra questa formulazione, come si vede esattamente sovrapponibile a quella goldoniana da cui abbiamo preso le mosse:

...el principal intento que las repúblicas bien ordenadas tienen permitiendo que se hagan públicas comedias es para entretener la comunidad con alguna honesta recreación, y divertirla a veces de los malos humores que suele engendrar la ociosidad...

Questa affermazione, relativa al pubblico bene, si colloca in un contesto molto più ampio, che merita una citazione più estesa, dedicato appunto a la buena comedia:

A lo cual respondería yo que este fin se conseguiría mucho mejor, sin comparación alguna, con las comedias buenas que con las no tales; porque de haber oído la comedia artificiosa y bien ordenada, saldría el oyente alegre con las burlas, enseñado con las veras, admirado de los sucesos, discreto con las razones, advertido con los embustes, sagaz con los ejemplos, airado contra el vicio y enamorado de la virtud; que todos estos afectos ha de despertar la buena comedia en el ánimo del que la escuchare, por rústico y torpe que sea, y de toda imposibilidad es imposible dejar de alegrar y entretener, satisfacer y contentar, la comedia que todas estas partes tuviere mucho más que aquella que careciere dellas, como por la mayor parte carecen éstas que de ordinario agora se representan. Y no tienen la culpa desto los poetas que las componen, porque algunos hay dellos que conocen muy bien en lo que yerran, y saben extremadamente lo que deben hacer; pero como las comedias se han hecho mercadería vendible, dicen, y dicen verdad, que los representantes no se las comprarían si no fuesen de aquel jaez; y así, el poeta procura acomodarse con lo que el representante que le ha de pagar su obra le pide. Y que esto sea verdad véase por muchas e infinitas comedias que ha compuesto un felicísimo ingenio destos reinos, con tanta gala, con tanto donaire, con tan elegante verso, con tan buenas razones, con tan graves sentencias, y, finalmente, tan llenas de elocución y alteza de estilo, que tiene lleno el mundo de su fama; $\mathrm{y}$, por querer acomodarse al gusto de los representantes, no han llegado todas, como han llegado algunas, al punto de la perfección que requieren.

L'ottica del «ben regolato governo» secondo le istanze «riformatrici» che sostiene la particolare accezione «repubblicana» (e veneziana) del fuoco della dedica gol- 
doniana si ritrova, dunque, in un discorso sulla "commedia» di una delle più grandi e famose opere europee dell'età moderna, «romanzo» a plurimi cassetti. Qui república va ovviamente intesa, alla lettera, nel senso più ampio di «cosa pubblica» (e della república cristiana), e l'utilità del teatro ad essa probabilmente ribalta o aggiorna il giudizio di espulsione a suo tempo decretato da Platone, e, in ogni caso, nella Spagna dell'inizio del XVII secolo la sfera in cui si iscrive questo discorso è quella della concessione del Monarca (e dell'autorità religiosa). Si tratta, certo, di un luogo stereotipo e via via codificato, che andrà a confluire in quello, tutto settecentesco e positivo, di repubblica letteraria (per la Spagna si veda, ad esempio, utilmente interrogabile nelle concordanze in rete, La poética o reglas de la poesía en general y de sus principales especies di Ignacio de Luzán, 1737). ${ }^{5}$

Si consideri qualche altro riscontro nel Quijote, mettendone da parte uno, su cui torneremo in chiusa, dove si vede che la questione dei romanzi cavallereschi si intreccia a quelle del gioco e dello spettacolo, dalla licenza all'utilità, fino a toccare le basi stesse su cui la "società» riposa, ovvero le istituzioni delle armi e delle leggi:

Ya os he dicho, amigo — replicó el cura—, que ello se hace para entretener nuestros ociosos pensamientos; y así como se consiente en las repúblicas bien concertadas que haya juegos de ajedrez, de pelota y de trucos, para entretener a algunos que ni quieren, ni deben, ni pueden trabajar, así se consiente imprimir y que haya tales libros, creyendo, como es verdad, que no ha de haber alguno tan ignorante, que tenga por historia verdadera ninguna destos libros. (I, 32)

A esto responden las armas que las leyes no se podrán sustentar sin ellas, porque con las armas se defienden las repúblicas, se conservan los reinos, se guardan las ciudades, se aseguran los caminos, se despejan los mares de cosarios, y, finalmente, si por ellas no fuese, las repúblicas, los reinos, las monarquías, las ciudades, los caminos de mar y tierra estarían sujetos al rigor y a la confusión que trae consigo la guerra el tiempo que dura y tiene licencia de usar de sus previlegios y de sus fuerzas. (I, 38)

Verdaderamente, señor cura, yo hallo por mi cuenta que son perjudiciales en la república estos que llaman libros de caballerías. (I, 47)

Quanto ai libros de caballerías, e posto che abbiamo incrociato all'inizio il nome del marchese Maffei, sarebbe anche da chiedersi dove la questione dei roman-

5. Al sito http://www.cervantesvirtual.com. 
zi termini e dove cominci quella del codice cavalleresco, nel senso del fondamento feudale, ovvero dove la biblioteca di Don Ferrante cominici a rappresentare un'altra collezione, diversamente "superata», rispetto alla biblioteca di Don Quijote. Ma questo è un argomento da meditare, eventualmente, altrove.

Mi importa qui, più che una dimostrazione di un possibile o probabile rapporto diretto tra la tirata cervantina e il passo goldoniano, senza entrare nel merito di possibili fonti ancora anteriori alla prima o di riprese intermedie, il riconoscimento di una tradizione culturale di ampia portata di cui, dati due punti, si può disegnare una linea o un asse: quella che chiamerò il discorso sulla buona commedia.

In ogni caso, inseguendo le occorrenze della parola republica nel Quijote si ricava che la buena comedia — come le armi e le leggi- risulta adorno de las repúblicas e che las repúblicas possono dirsi bien concertadas o bien ordenadas anche attraverso la sua legittima e regolata (controllata dalla autorità) funzione. Ma il discorso di Cervantes ha un altro fuoco essenziale e imprescindibile, ben presente, anzi centrale, in altre pagine del commediografo veneziano e ricorrente di testo in testo. Quello che Cervantes, appunto, definisce in termini di chiarezza estrema nella seguente dichiarazione: las comedias se han hecho mercadería vendible. E tirando in ballo il più grande, prolifico, fortunato e remunerato drammaturgo «per la scena» del suo tempo, ovvero Lope de Vega. Il discorso sulla buona commedia - ecco il puntosi caratterizza nella tradizione del teatro commerciale europeo dell'età moderna come, al tempo stesso, il discorso sulla commedia come mercadería vendible.

Come è noto in una fitta letteratura critica il discorso sulla commedia posto quasi alla fine del primo Quijote è stato considerato come una perorazione a favore della commedia classicista e delle sue regole che un drammaturgo sfortunato e misconosciuto opporrebbe alla libertà senza cognizione del teatro commerciale, indicando in Lope il suo idolo polemico. Un'interpretazione che non regge per una serie differente di ragioni a una minima verifica, a partire dal giudizio qui espresso, ampiamente positivo, sull'operato di Lope, e senza tener conto della costruzione ambigua e aperta, del rapporto evidentemente non di semplice e totale identificazione tra le parole del curato e del canonico e il punto di vista dell'autore (chi, peraltro, Miguel de Cervantes in persona propria o Cide Hamete Benengeli, il cronista arabo da cui egli finge di attingere storie e discorsi?). Desumere una secca determinazione ideologica per la commedia sarebbe come ricavare dal contesto più ampio, dedicato ai libri di cavalleria, che Cervantes era un avversatore del romanzo 
cavalleresco, mentre tutto quello che egli dice o racconta, dopo i primi capitoli, va messo a carico del fittizio sguardo estraniato dell'arabo che ha raccolto le vestigia della storia. Tra le ragioni di dettaglio si danno l'ampio e positivo giudizio quand'anche non sprovvisto di invidia - che vede la chiamata in causa del grande Lope e, come ora è stato persuasivamente mostrato in rapporto al teatro cervantino e alle dichiarazioni di poetica qui offerte, in particolare dal fatto che aperta risulterebbe la contraddizione tra ciò che Cervantes predica nella prima parte del Quijote e ciò che egli ha realizzato nelle sue otto comedias e negli altri testi teatrali, perduti, composti nel corso della sua intera esistenza.

In una rilettura in contropelo del capitolo del Quijote - parallelamente alla grande impresa dell'edizione del teatro cervantino per la Real Academia- ${ }^{6}$ Valle Ojeda ha osservato un vistoso errore di partenza dell'inquadramento tradizionale della questione:

[...] se da por supuesto que cuando el cura y canónigo hablan de arte, este se identifica con el arte clasicista y con la teoría dramática desarrollada por el neoaristotelismo italiano del siglo xvi. Y aquí radica, a mi parecer, el error de partida. $^{7}$

Le opere che rappresentano la buena comedia, citate dal canonico, non possono infatti dirsi «classiciste» o ispirate alle regole, posta la mescolanza di genere e la libertà di escursione nello spazio e nel tempo che presentano: Isabela, Filis y Alejandra di Lupercio Leonardo de Argensola, La ingratitud vengada di Lope de Vega, la Numancia dello stesso Miguel de Cervantes, El mercader amante di Gaspar Aguilar e La enemiga favorable di Francisco Agustín de Tárrega mostrano quanto sia fuorviante la riconduzione del discorso cervantino all'orizzonte aristotelico e classicista.

Ne emerge anche, e soprattutto, cosa che dovrebbe essere ovvia e che non lo è, che la dizione di comedias riguarda non il genere inteso nel senso della tradizione aristotelica italiana del secondo Cinquecento, ma definisce in generale le «opere drammatiche» e nella particolare pratica del teatro commerciale. Ne risulta

6. Miguel de Cervantes, Comedias y tragedias, e si veda in essa, nel volume di complemento, Gómez Canseco e Ojeda Calvo [2015:15-24] (anche con la documentazione di fonti italiane nel discorso poetico cervantino, tra Ludovico Dolce e Giovan Battista Giraldi, ovviamente al di qua del porsi della questione di un teatro a statuto commerciale).

7. Ojeda Calvo [2016:340]. 
quindi — posta la differente giurisdizione della Comedia-che anche la parola arte è qui investita sostanzialmente del medesimo significato che attraversa l'intera cultura europea dell'epoca. Vorrei mostrare, in una ricerca più ampia e a partire da fondamenti dell'Ars poetica oraziana, come questo orizzonte non risulti niente affatto riducibile al campo della cosiddetta determinazione classicista, come si crede. Ars (poetica) è parola che nella lettera ai Pisoni nemmeno appare - posto che il titolo spetta a Quintiliano- e che si ritrova in particolare, pochi anni dopo la pubblicazione del primo Quijote, nella sua formulazione più famosa, memorabile e investita di funzione militante, proprio nel titolo di Arte nuevo de hacer comedias.

Non so - e almeno non mi pare - che sia stata considerata un'influenza, o quanto meno un'intersezione, dei discorsi di Cervantes e di Lope nel senso di una comune, rinnovata, determinazione proprio della parola arte, dove la regola principale della buena comedia risiede in un sapere drammaturgico che si riporti positivamente, tramite il controllo del poeta, ai diritti di libertà della scena moderna e del gusto del pubblico (semmai l'orizzonte polemico riguarda le riprese indebite e le metamorfosi dei testi del «poeta» nella pratica degli autores de comedias, cioè i capocomici). Non si tratta della messa in campo -in opposizione a Lope e al teatro commerciale- di una precettistica classicista, ma piuttosto del richiamo alle prerogative distintive del drammaturgo e al suo (ideale) diritto di esercizio. Come potrebbe, del resto, l'autore del Rufián dichoso e altre comedias appellarsi ad essa?

Certamente Cervantes può aver invidiato la diversa fortuna di Lope, certamente il discorso di Lope si mostra più ardito e aperto nel rapporto con la cosiddetta "precettistica" e nella perdita di rispetto per Aristotele (mentre però si nutre della lettura dei neoaristotelici, come Robortello), ma i due discorsi (e consideriamo ovviamente in quello cervantino anche le formulazioni offerte dalla Comedia personificata all'inizio della seconda jornada del Rufián dichoso) rivendicano comunemente la centralità del ruolo e del controllo nel senso dell'arte esercitato proprio da coloro che Cervantes definisce los poetas que las componen.

Las comedias se han hecho mercadería vendible: ecco, come abbiamo detto, il crinale distintivo della questione, per come essa si pone dalla seconda metà del XVI secolo, prima in Italia e poi nell'Europa intera, con la nascita di un teatro commerciale. Ed ecco, al tempo stesso, l'elemento determinante l'esigenza di nuove (ma 
preferirei dire differenti, posta la sostanziale aderenza ad Orazio) poetiche, concepite in relazione, o in soluzione di compromesso, tra dignità letteraria e gusto del pubblico. I nuovi poeti teatrali sono coloro che non solo non possono essere incolpati delle licenze estreme dovute all'assenza di cultura dei praticanti senza cognizione che si cimentano con la scrittura drammatica (no tienen la culpa desto), ma questi poeti «responsabili» sono gli stessi che sanno quando, se costretti, errano concedendo troppo al gusto corrivo, perché conoscono ciò che vogliono o vorrebbero fare liberi dal giogo del commercio: porque algunos hay dellos que conocen muy bien en lo que yerran, y saben extremadamente lo que deben hacer.

In questa dialettica tra consapevolezza e adeguamento riposa - in fondo- lo stesso nesso della definizione (prevalentemente in realtà retrospettiva o ex post) della cosiddetta Riforma goldoniana: mentre il tragitto reale di svolgimento va dall'esperienza scenica alla riflessione teorica, la dichiarazione di "poetica» finge un piano preordinato, realizzato prudentemente, tappa dopo tappa, nella pratica.

Come si vede - e in una tradizione come quella italiana in cui la commedia all'improvviso mette diversamente al centro la figura dell'attore-autore - il raggio complessivo della difesa cervantina della buena comedia è il medesimo in cui si svolge ancora, un secolo e mezzo dopo, il discorso goldoniano sulla commedia, certo in termini storicamente e contestualmente mutati, ma sempre secondo la medesima triangolazione: il teatro come mercadería vendible; il ruolo del poeta teatrale e la sua «poetica»; l'orizzonte della república, in cui si inscrive ogni «riforma», tra onesto divertimento ricreativo e correzione finalizzata all'«utile pubblico». ${ }^{8}$

\section{Da Goldoni a Lope}

Nella prima illustrazione biografico-allegorica dell'edizione Pasquali, iniziata subito prima di trasferirsi a Parigi, il commediografo si fa rappresentare bambino, all'età di otto anni, in atto di scrivere la sua prima commedia, sotto lo sguardo della madre e di un precettore:

8. Per il panorama spagnolo, cfr. il classico Emilio Cotarelo y Mori [1904] e Marc Vitse [1990]. 
...principiando dall'età d'anni otto, in cui il genio comico principiava in me a svilupparsi, composta avendo in sì tenera età una commedia, di quel valore che aspettar si potea da un bambino. ${ }^{9}$

Goldoni conosceva ovviamente Lope e l'Arte nuevo, come si evince da citazioni sparse e da luoghi rilevanti, dove egli definisce la dignità della propria pratica della scrittura teatrale iscrivendola insieme in un regime di ars poetica e di attenzione e dipendenza dal pubblico giudizio e gradimento, e cioè da un carattere "commerciale» o di «mercato». Basti leggere quanto testimoniato dal primo dei suoi testi teorici, la Prefazione all'edizione Bettinelli del 1750:

Anche il gran Lopez de Vega, per testimonianza del medesimo scrittore, non si consigliava componendo le sue commedie con altri maestri che col gusto de' suoi uditori. ${ }^{10}$

Un carattere, in realtà — questo un punto che voglio sollevare- inscritto fin dal fondamento, nell'Ars poetica oraziana, in vari luoghi e soprattutto nel riferimento del poeta drammatico al cor spectantis (di un pubblico che mescolava, anche allora, come ai tempi suoi e nel siglo de oro spagnolo, equites peditesque), e, per esempio, nella raccomandazione al poeta drammatico di una condotta che metta insieme i dettami dell'ars della composizione e ciò che il pubblico desidera: «Tu, quid ego et populus mecum desideret, audi». Questo per prendere immediatamente distanza dall'idea di un «Orazio classicista», che detta norme generali per la poesia, confondendo il suo utilizzo moderno con il raggio storicamente accertato dell'Epistola ai Pisoni.

L'ars poetica di Goldoni è una commedia, non un trattato, e si intitola Il Teatro comico: una "poetica in azione», appunto, esattamente contemporanea alla prefazione Bettinelli, che aprì in forma di «prologo» drammatico, utilizzando e ampliando una consuetudine teatrale invalsa, la fatidica stagione delle cosiddette "sedici commedie nuove» dell'anno comico 1750-1751. Il personaggio del capocomico Ottavio (che rinviava al nome di scena del capocomico reale con cui Goldoni collaborava, ovvero l'autor de comedias, per dirlo alla spagnola, Girolamo Medebach) viene ribattezzato Orazio nella seconda edizione del testo, dopo che Goldoni passò a un'altra compagnia. Si tratta, insieme alla cancellazione fisica del capocomico associato

9. Goldoni, Tutte le opere, I, pp. 623-624.

10. Goldoni, Tutte le opere, I, p. 772. 
nell'impresa originale, di un evidente compimeneto del nucleo oraziano (messo a fuoco anche attraverso Lope), che riguarda una serie di meditazioni riprese proprio dai secolari argomenti che dall'epistola oraziana si dipartono (come, per esempio, il «Nec quarta loqui persona laboret» del v. 193, a proposito del fatto di non superare i tre interlocutori contemporaneamente in scena).

Chi contrappone la "classicità» di Orazio (specialmente dell'Orazio dei commentatori neo-aristotelici italiani del xVI secolo) alla «modernità» di Lope, come spesso si fa, dimentica però due fatti: primo che un'assunzione dell'Ars poetica in un senso classicista di precettista generale per la letteratura tutta non è un carattere originale né domina l'intera tradizione occidentale; secondo che il montaggio di argomenti consentito dalla forma libera e aperta dell'epistola (ciò che Lope, da par suo, ha compreso, riprendendo la forma in versi) è esattamente il tratto essenziale della tradizione della poetica drammatica (e si rammenti: Orazio si leggeva e commentava nel Medioevo, quando la Poetica di Aristotele era un oggetto del tutto assente o incompreso). La mossa goldoniana della "poetica in atto" ne rappresenta una prosecuzione tanto inedita e profondamente rinnovata quanto sostanzialmente concorde ai «fondamenti» del discorso: l'Ars poetica è, infatti, originalmente un trattato dedicato all'ars drammatica e rappresentativa, comprendendo l'epica in questo campo in quanto genere «recitato» ad alta voce davanti al pubblico.

Ma torniamo a Goldoni bambino allo scrittoio. Ora, era proprio ne l'Arte nuevo che Goldoni poteva trovare un esempio eloquente di autore che racconta il proprio rapporto con la tradizione che lo precede, e che egli supera e reinventa baldanzosamente, raccontato come quello di un bambino, "genio» precoce, che si misura con una drammaturgia "bambina», appena uscita dalle fasce, di un teatro che non ha ancora raggiunto la posizione eretta (l'esercizio di applicazione alla drammaturgia era peraltro, specialamente nei collegi dei padri gesuiti, come mostra la pratica goldoniana, un esercizio dell'età di formazione).

Sulla questione specifica della divisione in atti del dramma che è al centro del passo di Lope ("andaba en cuatro, como pies de niño») ho discusso ampiamente altrove, ${ }^{11}$ ponendo la questione su un piano complessivamente europeo, mentre qui vorrei semplicemente richiamare il senso generale — di tradizione - che marca il passaggio dalle forme precoci alle forme investite dalla maturazione d'autore:

11. Vescovo [2007: in part. pp.114-182]. 
[...] que eran entonces niñas las comedias;

y yo las escribí, de once y doce años (vv. 218-219)

Ecco, dunque, se l'accostamento è proponibile, come credo, una conseguenza decisiva o uno spostamento sostanziale. Sui dorsi dei volumi, del tutto ideali, della sua «biblioteca di quel tempo», che il Goldoni maturo immagina alle spalle di sé bambino allo scrittoio, si leggono prevalentemente nomi di scrittori italiani di teatro del XVIII secolo (FAGIUOLI-MAGGI-LEMENE-GIGLI), nomi che coincidono con quelli che si identificano solitamente come i precursori o i battistrada della cosiddetta «Riforma» goldoniana, intesa in questo caso non nel senso delle armoniche "politiche» alle riforme della "Repubblica», ma nel senso del recupero della dignità della scrittura drammatica contro lo strapotere dei comici e il primato italiano dell'improvviso. Drammaturghi da cui Goldoni — nel testo che accompagna questa immagine e in altri luoghi- prende le distanze, dichiarando di non avere debiti nei loro riguardi e mostrando scarsa considerazione per essi, certo non senza cattiva coscienza e rimozione di crediti. E del resto Goldoni non ha messo in libreria gli autori a lui vicini e davvero importanti, come Pietro Metastasio o Pier Jacopo Martello. ${ }^{12}$

Si suole considerare tradizionalmente (ma, grazie a Dio, i latinisti di solito non lo fanno) la lettera ai Pisoni, come ho già ricordato, un trattato di "poetica», dedicato al campo dei generi letterari in generale. Le Artes poeticae del classicismo ne ampliano il campo e, di conseguenza, ne disperdono il significato primo, specie se nell'unione o nella rilettura alla luce di Aristotele (particolarmente significativo qui il ruolo di un trattato ben noto allo stesso Lope, quello di Francesco Robortello) per la fondazione di una "poetica» a tutto campo, dove Orazio assume un ruolo di appendice o satellite. ${ }^{13}$ Un significato che torna invece esattamente al centro nella linea Lope-Goldoni, accomunati dal fatto di essere scrittori di commedie destinate al commercio: non autores de comedias come il capocomico Ottavio (poi appunto trasformato in Orazio) nel Teatro comico, ma ingenios che guardavano insieme alla dignità drammatica e al pubblico.

12. Della questione ho discusso in La biblioteca di Carletto Goldoni (Vescovo 2016a).

13. Da distinguere, ovviamente, l'Ars poetica di Marco Girolamo Vida, di generico reinvestimento «umanistico», come raccolta di consigli del precettore all'allievo, rilevante però per la ripresa (in latino) della struttura in versi: una linea che accomuna in fondo, dal puro punto di vista formale, l'esperienza seicentesca di Lope e Boileau. 
Sorpendente è stata per me recentemente — per questa e per altre ricerchela lettura dei commenti di data più altra, talora altissima, all'Ars poetica, che mi hanno messo di fronte a un'insospettata continuità di tradizione. ${ }^{14}$ Un discorso sui commenti medievali all'Ars poetica di Orazio — alcuni, di provenienza francese, diffusissimi in Europa- non può essere fatto in questa sede: tra i compiti di questa ricerca, tutta da compiere, c'è ovviamente, tra gli altri, quello di stabilire, nei limiti del possibile, l'estensione e la continuità di un sapere di lunga durata sulle cui basi si rifonda la storia stessa del teatro moderno. Resta da capire e indagare se e come le questioni che appaiono chiarissime nella tradizione dei commenti medievali si tramandino nella cultura europea, soprattutto scolastica, dei secoli seguenti, posta la conservatività di questa, e come attraverso questa tradizione si trasmetta il significato originale dell'Ars poetica, cioè quello di un discorso specifico sui generi drammatici e sul rapporto col pubblico: vale a dire esattamente quello che si perde nel raggio delle poetiche classiciste. ${ }^{15}$

Non c'è dubbio, in ogni caso e tornando al nostro argomento, che l' Arte nuevo

14. Per un panorama complessivo dei commenti orazioni e per un progetto di ricerca di grande utilità per la questione cfr. http://www.univ-paris3.fr/index-des-commentateurs-a-g-80490.kjsp?RH $=1275913194592$.

15. Accenno rapidamente a una questione, tra le tante che non ho qui modo qui di affrontare: quella del rapporto di Lope con Robortello, che riguarda il peso della tradizione oraziana nel suo trattato. Come è noto Lope si serve abbondantemente della terza explicatio annessa alla Poetica di Robortello, quella dedicata alla commedia, e citando direttamente la sua fonte (ciò che è puntualmente annotato fin dall'edizione dell'Arte nuevo di Juana de José Prades). Resta da indagare, mi sembra, cosa e dove abbia eventualmente Lope attinto dalla parte principale del trattato e dagli altri annessi. Personalmente mi sembra siano visibili alcune tracce di un impiego più ampio, ma la questione richiede applicazione di dettaglio. Credo invece - almeno per quanto evidente a un livello di osservazione di massima- che ininfluente, o scarsamente influente, risulti per Lope l'explicatio dedicata all'Ars poetica oraziana. Come può essere che un testo profondamente oraziano come l'Arte nuevo - che, fin dal titolo, mostra la sua linea d'inquadramento e di cimento nel rinnovamento e che palesemente mutua dall'epistola in versi la forma del trattato aperto e non sistematico- non ne tenga conto? La mia risposta è doppia e le sue due componenti — una nel testo di Robortello e una nella tradizione- si combinano. Nel primo, l'orizzonte del commentatore esclude del tutto e sistematicamente le pendenze - strutturali e determinanti- di cultura materiale del teatro presenti nell'epistola oraziana (si parla perfino del sipario, della divisione in atti e delle tibie suonate nell'intervallo, oltre che di generi, di stili, di determinazione del linguaggio dei personaggi, eccetera). Le aggira, anzi, spesso con parafrasi che distolgono dal senso letterale: Orazio parla, per riassumere sinteticamente, di spettatori a teatro, Robortello di lettori a tavolino di dramma e poema epico. Nella tradizione - come ho già detto (questo è il mio sujeto) - la lettera ai Pisoni è stata letta, anche nel medioevo e nella tradizione scolastica successiva, come un compendio dell'ars drammatica (comprendente l'epica) destinata alla rappresentazione. Ciò che sparisce, appunto, quando l'Ars poetica è piegata a diventare un satellite della Poetica aristotelica. 
di Lope si ponga come una nuova Ars poetica per la scena, in una tradizione sicuramente e profondamente oraziana, che riguarda i generi rappresentativi, tra l'arte della composizione poetica e l'orizzonte del pubblico.

\section{ARS COMICA E COMMEDIA DELL'ARTE}

Si possono accostare le categorie di Ars poetica - nel senso fin qui indicato (senso originale e sulla cui lunga tradizione torneremo a dire) di Ars drammatica o Ars comica-e di Commedia dell'arte? Si tratta di un'inversione di termini significativa o di un'apparente omonimia dislocata?

Nell'etichetta commedia dell'arte la parola arte significa indubbiamente «corporazione professionale», come si chiamavano arti quella dei sarti o dei carpentieri. Il suo rapporto con l'altro ed esteso uso di arte - che incontriamo per il teatro e i generi drammatici appunto nell'Ars oraziana, nel Quijote e nell'Arte di Lope- non mi sembra essere stato considerato. Tentare questa via non significa tornare a letture fuorvianti (celebre quella di Allardyce Nicoll, di arte intesa come «teatro fatto da attori di singolare talento»), ma risalire un cammino storico di lunga durata e complessità.

Una rivendicazione chiarissima di una distinzione dal genere commedia inteso secondo tradizione neoaristotelica in ragione di un'assunzione dell'ars drammatica da parte dei comici di mestiere si trova nelle righe che definiscono il contenuto de la Supplica di Niccolò Barbieri (1636). Si rammenti che Lope - come del resto in futuro Goldoni- non calcava la scena, e Barbieri, Cecchini ed Andreini, come del resto Molière, sì.

La Supplica, dove discorso famigliare diventa sottotitolo, rappresenta la forma ampliata —come recita il frontespizio - dell'originale Discorso familiare intorno alle moderne commedie (1628), il cui titolo riprende un'esattissima dizione lopiana, quella di comedia nueva. Prima di citare il celebre passo, riporterei un paio di righe in cui Barbieri manifesta in termini particolarmente attenti l'inversione del rapporto del dare e dell'avere tra Italia e Spagna dal punto di vista della circolazione della mercanzia teatrale: 
La Spagna prima si serviva delle nostre italiane e i comici vi facevano assai bene; Arlicchino, Ganassa e altri hanno servito la felice memoria di Filippo secondo e si fecero ricchi, ma dopo quel Regno ne ha partorite tante che ne riempie tutti quei gran paesi e ne manda anche molte Compagnie in Italia. ${ }^{16}$

Ci basta ai fini della dimostrazione, mentre il frontespizo della prima redazione parla da solo. E si legga l'attacco della Supplica:

Ragionando della Comedia non intendo trattare del Poema diffinito da Orazio, Aristotele ed altri per imitazione d'azzione umana e perfetta ma non illustre, la quale dee ravvolgersi e terminarsi nello spazio d'un giorno, attendendo sempre al publico giovamento, chiudendo la sua catastrofe in giocondità, lasciando lo scettro alla Tragedia sua maggiore sorella intenta a più alti documenti ch'alla volgar cura, ma intendo parlare della mia professione difendendola per come saprò, mostrando a chi è mal informato dell'esser suo che non è vile, né scandalosa come tali la dichiarano; per tanto sotto questa voce di comedia voglio sempre inferire l'Arte in genere, qual rappresenta tanto Comedie quanto Tragedie, Pastorali, Tragicomedie, Pescatorie ed altr'opere miste, atteso che noi rappresentiamo istorie e favole, intrecciando le cose serie fra le giocose, per non render nel corso d'una stagione sazietà ne' gusti, e far manchevole, co' gusti di color che n'ascoltano, l'utile nostro. ${ }^{17}$

Il passo ha alle spalle diverse tradizioni e mette in campo alcuni slittamenti (voluti o ereditati) dall'uno all'altro piano: da quello della poetica (Ars comica appunto come "arte drammatica", nel rapporto di distinzione ma anche di ripresa dal campo designato nell'Ars poetica oraziana) a quello della pratica rappresentativa (l'Arte drammatica esercitata dai comici di professione).

Certamente chiaro — si tratta di una dichiarazione d'ingresso- risulta il panorama da cui Barbieri vuole prendere distanza: dal significato ristretto che la parola commedia ha assunto nella tradizione delle poetiche dedicate prevalentemente al genere drammatico, soprattutto nella trattatistica aristotelica recente. Ma andrà ricordato che commedia -lungi dalle partizioni di genere, certo discendenti da Aristotele e certo presenti in Orazio e investite dalle Poetiche moderne (in particolare neoaristoteliche e della linea Boileau) — è nei secoli XVII e XVIII termine onnicom-

16. Si veda l'edizione a cura di Ferdinando Taviani, La supplica, p. 89.

17. Barbieri, La supplica, ed. Taviani, pp. 60-61. 
prensivo per la definizione del genere drammatico, come appunto Barbieri dettaglia in una dichiarazione relativa all'ampiezza dei generi nel repertorio scenico.

Questo è il senso che assume la categoria di comedias di Lope, a cui si riferisce l'altra giurisdizione di un Arte «nuevo», e questa l'estensione dell'aggettivo comico che accompagna più di un secolo dopo nella «poetica in azione» goldoniana il sostantivo Teatro. Quel Teatro comico non considera infatti (almeno non principalmente) la giurisdizione della commedia, ma del teatro esercitato dai comici, considerando il ruolo del poeta teatrale (che, infatti, non appare in scena, o vi appare solo nel doppio negativo del "poetastro» Lelio, che finirà infatti con l'essere assunto dalla compagnia in prova come attore).$^{18}$ Del resto anche la lingua francese del «tempo delle regole» di Boileau e della tradizione neoaristotelica chiama comédiens gli attori (i comici, secondo la definizione tramontata nel lessico italiano e i comediantes spagnoli) e Comédie l'istituzione teatrale (quali, per esempio, la Comédie italienne e soprattutto la Comédie française).

Per contro arte ha una doppia, ovvia, accezione: quello di categoria o gruppo professionale e quella di pratica da esso esercitata e che individua un sapere tecnico e un esercizio di professione: Barbieri parla infatti di artieri e di artegiani quando fa riferimento alle arti meccaniche propriamente intese (mentre arte evolve verso l'accezione, che ci è comune, nella distinzione che stacca l'artista dall'artigiano). I comici si definiscono, insieme, come una corporazione professionale di «non meccanici» e l'arte comica - ovvero la loro specialità o tecnica distintiva- si offre come terreno di complessa determinazione, che oscilla tra un esercizio della scrittura e un esercizio di quello che possiamo sbrigativamente definire il mestiere della scena (e che assorbe, eventualmente, nella tradizione italiana, posta la caratterizzazione specifica dell'improvviso, la "scrittura in scena»). ${ }^{19}$

Qual'è il carattere specifico che si profila rispetto alla tradizione oraziana dell'ars drammatica nell'età in cui i comici, come Cecchini, Barbieri, Andreini, scrivono di questi temi? Ovviamente la costituzione organizzativa di un teatro commerciale, con la definizione morale e legale di tale impresa. E potremmo riassumere in maniera centonaria: non la «tecnica» della corporazione (l'esercizio del «mestiere»), né la definzione della scrittura per la scena (ciò a cui si dedica lo stesso Lope) sono

18. Cfr. Vescovo [2012].

19. Per una precisa rilettura in questo senso dell'esperienza goldoniana —uno «poeta» che si inserisce nel sistema della cultura artigianale degli attori—cfr. le indicazioni di Siro Ferrone [2011]. 
l'oggetto di questo discorso e del formularsi di un suo ordine (nel senso che dava Foucault a questa parola nel celeberrimo L'ordre du discours, di tradizione che comprende ed esclude normando), ma qualcosa che non è né l'una né l'altra cosa, e che appartiene dunque alla sfera morale e giuridica che individua la liceità di una pratica e della sua organizzazione in mestiere.

Commedia significa appunto ed esattamente per Barbieri nel passo citato, come nei luoghi di poco precedenti di Cervantes e Lope, non la definizione di un genere normato retoricamente o secondo i dettami della poetica (l'oggetto specifico di applicazione della tradizione neoaristotelica, su questo l'autore è chiarissimo), ma l'Arte in generale, ovvero il raggio complessivo dell'Ars poetica - un testo che parla solo dei generi drammatici (ivi compresa l'epica recitata)—, qui considerata dal punto di vista nuovo e differenziante della professione comica: ciò che i comici di mestiere esercitano, ovvero una Arte comica, che comprende anche la scrittura (e quel particolare tipo di «scrittura» che si ingloba nella categoria dell'improvviso). Si veda la corrispondenza: l'Arte in genere rappresenta / noi rappresentiamo, dove il secondo termine è, e solo, indubbiamente riferito all'alternanza di offerta dei "generi» (di testo o di spettacolo) nel repertorio delle compagnie comiche. E da Cervantes e Lope a Goldoni si tratta, appunto, dell'orizzonte assunto per la difesa e la polemica dal drammaturgo che scrive per le compagnie ma che non calca direttamente la scena.

Uno spoglio dei passi della Supplica in cui appare la parola Arte mostra che l'accezione di «corporazione professionale» è, quanto meno, secondaria e difficilmente circoscrivibile rispetto a quello fondante di "pratica» (nel senso delle Arti liberali e meccaniche). ${ }^{20}$ Il cenno alla giusta mercede mostra un'indubitabile riferimento a San Tommaso (del resto ampiamente ed esplicitamente citato da Pier Maria Cecchini) e apre a una collocazione estremamente precisa il senso prevalente dell'impiego del termine Arte (Comica), appunto: non avvilita dalla corresponsione della mercede e per ciò indipendente da essa (anche l'equiparazione di San Cipriano di ars comica e ars magica è dibattuto da Cecchini, che è fonte evidente di Barbieri).

Il significato di Ars risale, sostanzialmente, alla tradizione scolastica e alla

20. Cfr. lo spoglio offerto in appendice. 
categoria di «causa strumentale» (causa instrumentalis). ${ }^{21}$ Questa la cultura che nutre la Supplica, e prima Cecchini, attraverso le riprese dei secoli seguenti: e non è un caso che centrale risulti il riferimento alle autorità e alla letteratura religiose, qui come in altri interventi (si pensi ancora a Giovan Battista Andreini), ${ }^{22}$ dove tanto la questione della «causa strumentale» quanto quella della liceità dell'azione e del ruolo dell'istrione si mostrano come la vera "archeologia» (sempre nel senso di Foucault) delle presenti difese. La Supplica media, in un terreno complesso e in larga parte non esplorato, i termini distanti con cui si dibatteva della circoscrivibilità di un'arte comica, di una pratica strumentale e di una «liceità di mercede», nel sistema evidentemente più complesso, rispetto a quello che fu degli istrioni, del teatro commerciale e della costituzione delle "compagnie» a partire dalla fine del XVI secolo. E riporta su questo terreno, per via complessa, il raggio dell'Ars poetica, riconsiderato dal rapporto poeta-recitator con cui lo poneva Orazio in quello di una Ars comica, quale patrimonio di una professione né "mercenaria» (nel senso deteriore del termine), né quale esercizio d'inganno o di pratica diabolica (ciò che spiega i fittissimi, nella nostra ottica altrimenti incomprensibili, riferimenenti che distinguono Ars magica da Ars comica).

Uno spoglio di Cecchini di Barbieri e di altri comici-intellettuali del primo XVII secolo - il secolo di Lope e di Boileau, «continuatori di Orazio» su sponde nettamente divaricate - non offre la documentazione della nascita o dell'anticipazione di una designazione di repertorio, ma piuttosto la rivendicazione coraggiosa della pratica o professione di una Ars. Presumibilmente solo nel corso del secolo seguente si attesta nella tradizione italiana un'etichetta, certo in origine di impiego gergale, quella di commedie dell'arte, che riguarda le pièces di un repertorio diffuso e la cristallizzazione e chiusura di quest'ultimo (essenzialmente «comico» nel senso tradizionale). ${ }^{23}$

21. L'Ars theatrica è registrata, infatti, da Ugo da San Vittore, «curiosamente» come annota, in margine di un densissimo discorso sulle «tecnologie strumentali», Giorgio Agamben [2014:105]: «E -curiosamente-gli spettacoli».

22. Si veda il recente volume Giovan Battista Andreini, Opere teoriche, a cura di Rossella Palmieri.

23. Per una trattazione più ampia della questione mi permetto di rinviare al mio La commedia dell'Arte (Vescovo 2012). È stato recentemente sostenuto che nella Supplica si riscontrerebbe l'anticipazione dell'uso, certo gergale e che vediamo diffondersi da metà Settecento, della categoria delle commedie dell'arte (poi al singolare commedia dell'arte), riferito alle commedie del repertorio comico diffuso: in realtà un solo passo sembra piegabile in questa direzione ("e pur la più debole comedia che sia nell'arte è più profittevole di qual si voglia altro trattenimento»: p. 84), che può indicare, al massimo, il nucleo remoto di un'idea di appartenenza dei «testi» o delle pièces rappresentate al gruppo professionale. Ma la storia di questa lenta emersione nell'idea di essere nell'arte o di appartenere 
Ovviamente le compagnie dell'arte continuavano a recitare altro oltre alle commedie con le maschere - quelle che diventeranno le commedie dell'arte, con una restrizione di campo che data alla sclerotizzazione di quel repertorio- e la designazione riguarda, dunque, solo una zona diffusa ma conservativa di una tradizione: «se facciamo le solite commedie dell'arte vogliamo star bene!», dice la primadonna del Teatro comico. È qui, su questo fronte e in questi termini, che Goldoni interviene e tenta una sua nuova Ars poetica (avendo presente soprattutto Lope, tra i continuatori di Orazio), che si distingue dalle altre per essere una "poetica in azione». Una poetica non solo in forma di commedia, ma fatta recitare originalmente ai suoi comici e, soprattutto, al suo capocomico. Facendo «rappresentare» (nel senso letterale e legale del termine) ad esso le istanze dell'autore.

\section{Progetto e ciarlatanerìa}

Come abbiamo visto nella rapida rassegna cervantina di partenza, non solo la lettura dei romanzi, il gioco e gli spettacoli (e dunque le comedias) sono dichiarate, anche se attraverso un inquadramento ironico, servire all'ordine della República, attraverso la distensione e la ricreazione, se non con scopi più alti di correzione del costume, accanto alle leggi e alle armi.

In un passo - che appartiene al più strampalato dibattere per via di don Quijote e di Sancho - si legge che addirittura il mestiere o la "carica» di alcahuete, cioè del ruffiano, rientra in tale novero, con evidente gusto dal paradosso. L'affermazione ricorre in ben due capitoli:

el oficio de alcahuete, que es oficio de discretos y necesarísimo en la república bien ordenada, y que no le debía ejercer sino gente muy bien nacida; [...] Quisiera pasar adelante y dar las razones por que convenía hacer elección de los que en la república habian de tener tan necesario oficio, pero no es el lugar acomodado para ello: algún día lo diré a quien lo pueda proveer y remediar (I, 22).

all'arte - che inscrive a un patrimonio collettivo un repertorio (differente e distintivo da quello dei «testi» precisamente acquistati da una singola compagnia) - merita, ovviamente, una ricerca specifica. Cfr. intanto in appendice le spoglio completo delle occorrenze del termine arte nella Supplica. 
y esta mesma regla corre por todos los más oficios o ejercicios de cuenta que sirven para adorno de las repúblicas (I, 25).

Un libro di Bartolo Anglani fresco di stampa — che raccoglie sì saggi già pubblicati, ma che ne rende evidente il complessivo spessore solo nella loro riunionesi dedica all'attrazione di Carlo Goldoni per le figure di ciarlatani, di avventurieri, di picari, mostrando la continuità e la pienezza di una dimensione che è, a questo punto, necessario comprendere essere l'altro fuoco - insieme a quello della progettualità organica o della Riforma - del discorso goldoniano. ${ }^{24}$ Se il secondo offre, soprattutto retrospettivamente, un ordine progettuale all'esperienza scenica, il primo ne rappresenta le spinte di partenza (non a caso centralissima nel racconto autobiografico), il nutrimento e l'ispirazione costante.

Ho altrove commentato a lungo — e non sarà qui il caso di tornare a riprendere la questione- l'episodio de L'avventuriere onorato, commedia di «allegoria» biografica che concludeva la stagione delle «sedici commedie nuove» (la stessa aperta dal Teatro comico), in cui il protagonista (che ha, ovviamente esercitato tra gli altri il mestiere del poeta teatrale), denunciato in quanto persona «incognita» e sospetta dai rivali, appare davanti al Viceré —l'azione si svolge a Palermo- per essere espulso con un foglio di via. Guglielmo reagisce prontamente, mostrando al sovrano come la mancanza di informazioni sul suo conto sia in realtà prova della carenza del sistema spionistico dello Stato e propone, su due piedi, un ricordo o un progetto di riforma del sistema, che gli varrà non solo il perdono ma l'offerta di una carica e di un riconoscimento economico. Ecco un esempio puntuale di come la ciarlataneria, nel senso della risoluzione immediata, o della sfrontatezza, possa accompagnarsi all'esibizione della progettualità. Qui —e in tutta questa commedia - Goldoni è in ogni particolare.

24. Anglani [2016]. Isolo un passo, tra i vari citabili, che riassume il modo, assolutamente rinnovato, di porre la questione: «Il ciarlatano è non tanto la traccia di un passato che resiste e a volte ritorna per ostacolare il progetto della Riforma, non è insomma il residuo passivo della Commedia dell'Arte destinato ad essere superato da una concezione e da una pratica più moderna della scena, ed è invece il polo sempre attivo di una contraddizione creativa senza la quale lo stesso progetto riformatore si ridurrebbe al catalogo delle proprie buone intenzioni. Goldoni sa bene che la traduzione dei temi potenzialmente drammatici in Teatro non può avvenire senza la mediazione dell'arte ciarlatanesca, anche quando quei temi sono profondamente «seri» e si propongono di analizzare la società da un punto di vista "progressivo». L'importante, per lui, è che quell' "arte» non sia gestita in autonomia dagli attori ma sia dominata dall'autore» (pp. 43-44). Il lettore vedrà da solo il ricorrere delle «parole-chiave»: arte, moderno, autore, attori, progetto, riforma. 
Il discorso sul libro di Anglani andrà ripreso e meditato, ma mi sia intanto permesso di impiegare «di sponda», brevemente, la proposta centrale che esso offre, per tornare alle questioni da cui siamo partiti. Che l'autore del Rufián dichoso -in cui un picaro e furfante arriva ad essere santificato - sia il medesimo che nel Quijote dichiara l'alchauete un oficio necesario a la República ben ordenada (aggiungendo, peraltro, che la funzione di ruffiano dovrebbe essere esercitata solo dai «ben nati»), non appartiene esclusivamente al campo del paradosso dilettevole. Scopre una verità, che è anche, forse soprattutto, la "verità" profonda a cui Goldoni spesso si avvicina: quella per cui riforma e ciarlateneria, come abbiamo appreso da Anglani, nella loro differenza combinata e nella produttiva ambiguità che esse generano, funzionano nel servizio alla República, negli ordini della «drammaturgia da vendere» e della drammaturgia da leggere, come le due dimensioni necessarie e ineliminabili di un contrasto teatralmente efficace e artisticamente produttivo. 


\title{
APPENDICE
}

\author{
Arte nella Supplica di Niccolò Barbieri \\ (IL NUMERO TRA PARENTESI RINVIA ALLA PAGINA DELL'EDIZIONE TAVIANI)
}

Dico dunque che l'arte comica è arte sempre d'un istesso nome, ma non sempre d'un istesso merito; e la diversità de' meriti non deriva dalla comedia, ma da' professori di tal essercizio [60: paragone con la pittura: la comedia è come una tavola da dipintore, qui drammatico dipintore]; arte del cantare (62); gli antichi comici s'inoltravano tanto nell'arbitrio, che non rappresentavano altro che oscenità, intrecciavano il sacro col profano, ed alle volte facevano azzioni in derisione de gli Ecclesiastici; professavano l'arte magica, come si cava da San Cipriano, bestemmiavano, in occasione di colera, il santo nome d'Iddio (64); Gran disaventura di quest'arte, che nel campo delle suevirtuose azzioni, più tosto le persone vogliono spigolare i biasimi che prender la ricca messe delle sue buone qualità (66); Ed ecco che forse l'invidia di tanti onori mosse tal uno a dir male di quest'arte (66); il far pianger le persone di cosa ch'ognuno sa non esser vera, è arte difficile (69); il fondamento dell'arte sua [del vasaio] è far vasi (70); Tali sono i comici virtuosi, che si sanno valere dell'occasioni e dell'arte (71); la verità fa lodar l'arte, ma non occulta il vizio (72); Io pensar altro non posso se non che la comedia fosse buonan per se stessa, ma che fosse professata da persone scostumate nell'arte e che perciò sbandita fosse (74); Non tutti vedono le compagnie de' galantuomini, né possono creder tanta onestàin quest'arte, avendo nell'idea una chimera che mostra loro la comedia per cosa impudica e i comici per indiscreti (76); In quest'arte è di mestiere un talento naturale, a pochi conceduto,e di cento che si pongono a recitare, dieci non riescono buoni (77); L'intenzione dell'arte è di giovare e dilettare, ove che i recitanti rimangono astretti per la natura dell'arte d'avanzarsi al giovamento, non potendo dilettare senza giovare, essendo che l'uno genera l'altro (77); E l'arte del descrivere le cose insegna mostrarle col loro contrario, per farle spiccar meglio (77); lo spenditore, che sa non esser la moneta buona, o la cambia o mostra con arte sdegnarsi (78); [gli spettatori che vanno a teatro solo per vedere le attrici] poco fastidio possono dar all'arte che non mira a tal fine (79); l'arte [medica] mira alla purga e non al danno (82); ma tu non intendi l'arte; molte volte si grida a terrore, ed ognuno fa l'offizio suo (83); Gran cosa che a certi la comedia dii più fastidio che 
ogn'altro passatempo; e pur la più debole comedia che sia nell'arte è più profittevole di qual si voglia altro trattenimento; ma forse dà noia a qualche persona l'arte del favellare, e forse ad altri il zelo della salute del prossimo gli muove, presupponendo rilassazione nell'arte o sinistro fine ne gli ascoltanti (84); l'arte che professa [l'orefice] è di conoscer i metalli (85); l'arte della comedia, quand'anche non portasse benefizio al prossimo, come tali pensano, [...] non si dovrebbe più ella dell'altre professioni in publico lacerare (86); artegiani (87); Se i comici servono Principi, gli servono per ricrearli e non per consigliarli, che non è arte loro (87); Quest'arte non si esercita senza permissione de' Superiori (89); parmi che mutar lo spasso vizioso in diletto civile sia arte da medicare senza apportar terrore d'annichilità (91-92); come que' grembiali o traverse che portano gli artegiani (92); la nobiltà oziosa e gli artefici poveri (92); mi pesa che certi impazienti alla distinzione dicano male dell'arte, che non v'ha colpa, e de' recitanti indifferentemente (94); non può esser altro che far conoscer a' comici come la loro salute sia quasi disperata, acciò che lasciano l'arte, o veramente spaventar le persone con minaccie d'Inferno se vanno alla comedia, acciò che la lasciano (94); i Superiori s'appigliano alla loro dottrina e che non permettano licenza di essercitare quest'arte (94); I comici non possono lasciar l'arte, poiché con tal professione campano (94); se la metà solamente di quello che scrivono io scorgessi esser vero, che lascierei or ora l'arte (95); L'arte è di sospetto (95); l'arte non capisce modi ingannevoli (96); e far che niuno più imparasse tal arte (96); Che non è bene confonder l'arte comica con le arti proibite (96); Dico che io non vorrei che questi autori di libretti confondessero, per far bene, l'arte comica con l'arte magica e dannarla con tal coperta (97); Il premio che si dà a' comici per le loro fatiche è giusta mercede ed il pagamento non avvilisce l'arte (98); Anzi, che i maggiori salarii, premi e doni, portano maggior onor all'arte (98); si pagano fino gli organisti e gli altri musici che s'affaticano più le feste che gli altri giorni, ancor che indirizzata sia l'arte loro a lodar Iddio (98); l'arte loro sarebbe finita mille anni sono (100); Che molti essercizii attendono alla destruzione della robba e della vita, cosa che non fa l'arte comica (100); L'arte comica non ha disposizione all'usure, alle vendite illecite, alle scritture false, a gli omicidi, né a gli inganni (101); Le comiche, quando parlano in iscena co' loro recitanti, non hanno tempo di girar con arte gli occhi per far preda de' cuori (102); Così l'uso del vedere le donne in comedia e l'udir i loro discorsi amorosi è un tal abito già fatto, per l'uso dell'arte, che non solletica così facilmente la concupiscenza, come tali si pensano (104); e si lasciano rapir dall'arte e non dalla libidine (104); non nasce né 
dall'odio né dall'amore che a' schermitori portano, ma dalla vaghezza dell'arte (104); l'intelletto gode dell'eccellenza de l'arte (104); Il papagallo [...] ha per arte il diletto (104); ognuno ha da far l'arte sua (105); e chi non ha tal arte, non conosce il costume dell'essercizio e non è buon rappresentante (105); i comici sogliano esercitar l'arte loro (107); per mostrare che l'arte va così (107); vi è differenza da chi ha per arte il furto a chi ha per fine guerriero onore (108); E forse ancora il benedetto Pastore non aveva piena cognizione dell'arte comica (108); rimaneva [il Bendetto Pastore] mortificato non poco d'aver mal trattato in publico l'arte comica e i professori (108); Che il benedetto Prelato non scrivesse contro comici virtuosi, o che non fosse ben capace dell'arte comica, io lo cavo da questo avvenimento (108); vanno poi quel giorno a trattenersi al giuoco, il quale talvolta è più faticoso che l'arte che professano, ma per esser tolto per ricreazione non sentono fatica (111); tal uno ha stimato che la comedia sii stata fatta ad arte per scoprir le sue disgrazie e i suoi eventi (112); Ma chi toglie impresa di lacerar quest'arte, non assottiglia la ragione per questo verso (113); se quest'arte del rappresentare non fosse lecita, Santa Chiesa la leverebbe afatto (114); Oltre ciò i comici chiedono licenza di far l'arte loro, e l'ottengono (114); l'arte del Calcante (117); Non apportar il vizio del comico fuori di scena detrimento all'arte (117); si leverebbe il vivere a' virtuosi artefici, ad industriosi mercatanti, ed a' poveri lavoratori (118); e quando ancora uno si servisse della comedia per istrumento a mali affari, la colpa non sarebbe dell'arte, ma de' mali operatori (119); Un saccente orefice che facesse moneta falsa, se ben la sottigliezza dell'arte e la comodità de' ferri gli fossero mezzani a far tal misfatto l'arte però non resta offesa (119); l'arte della medicina (119); ignorante nell'arte ch'ei professa (120); vi è differenza tra l'arte in spezie, e le persone in individuo (120); niuno si muove a far un'arte con pensiero di beneficare o danneggiar il prossimo (120); di mal trattar l'arte con simil coperta (121); far l'arte sua per vivere (122); Lo aver riguardo a' comici che recitano gratis e non a quelli che per necessità professano tal arte, è una carità da me non intesa od una giustizia da me non conosciuta (129); a' prieghi finti dell'arte (131); del frutto che apporta tal arte (132); pensa che, se tu fai l'arte tua, che gli altri fanno la loro (132); un comico recita come si deve e sta nel costume dell'arte (133); come il comico fa l'arte sua conforme il dovere (133); chi sono costoro e da dove deriva quest'arte e che ne dicano gli scrittori antichi (133), Che tutti gli autori che hanno scritto contro le comedie non hannoavuto l'intiera cognizione di quest'arte (134); è un'arte che mai avrà faccia di carità (135); l'arte della rettorica (135). 


\section{BIBLIOGRAFIA}

Agamben, Giorgio, L’uso dei corpi. Homo Sacer IV, 2, Neri Pozza, Vicenza, 2014.

Andreini, Giovan Battista, Opere teoriche, ed. R. Palmieri, Le Lettere, Firenze, 2013.

Anglani, Bartolo, Il "soave mestier della "birba"». I ciarlatani di Goldoni e altri saggi, Aracne, Ariccia, 2016.

BARBIERI, Nicolò, La supplica. Discorso famigliare a quelli che trattano de' comici, ed. F. Taviani, Il Polifilo, Milano, 1971; reed. Cuepress, Imola, 2015.

Cervantes, Miguel de, Comedias y tragedias, ed. L. Gómez Canseco, Real Academia Española, Madrid, 2015, 2 vols.

Cervantes, Miguel de, Don Quijote de la Mancha, dir. F. Rico, Galaxia Gutenberg-Círculo de Lectores-CECE, Barcelona, 2004, 2 vols.

Costantini, Giuseppe Antonio, Della Commedia italiana e delle sue regole ed attinenze, Giuseppe Bettinelli, Venezia, 1752.

Cotarelo y Mori, Emilio, Bibliografía de las controversias sobre la licitud del teatro en España, Madrid, 1904; reed. Universidad de Granada, Granda, 1997.

Ferrone, Siro, La vita e il teatro di Carlo Goldoni, Marsilio, Venezia, 2011.

Goldoni, Carlo, Tutte le opere, ed. G. Ortolani, Mondadori, Milano, I, 1935.

Gómez Canseco, Luis, y María del Valle Ojeda Calvo, "Cervantes y el teatro», in Miguel de Cervantes, Comedias y tragedias, ed. L. Gómez Canseco, Real Academia Española, Madrid, 2015, II, pp. 9-60.

Ojeda CALvo, María del Valle, «El engaño a los ojos y la teatralidad cervantina», in Geométrica explosión. Estudios de lengua y literatura en homenaje a René Lenarduzzi, eds. E. Sainz González et alii, Edizioni di Ca’ Foscari, Venezia, 2016, pp. 339-350.

Vega Carpio, Lope de, Arte nuevo de hacer comedias, ed. E. García Santo-Tomás, Cátedra, Madrid, 2006.

Vescovo, Piermario, Entracte. Drammaturgia del tempo, Marsilio, Venezia, 2007.

Vescovo, Piermario, «Guardando verso la scena. Il Teatro comico: una lettura», Studi Goldoniani, I n.s. (2012), pp. 11-25.

Vescovo, Piermario, "La commedia dell'Arte», in Il contributo italiano alla storia del pensiero. La letteratura, ed. Giulio Ferroni, Istituto dell'Enciclopedia Italiana, Roma, 2012, in corso di stampa. 
Vescovo, Piermario, «La biblioteca di Carletto Goldoni», in Goldoni «avant la lettre». Esperienze teatrali pregoldoniane (1650-1750), ed. J. Gutiérrez Carou, Lineadacqua edizioni, Venezia, 2016, pp. 667-678.

Vescovo, Piermario, "La felicità delle commedie», in Il "barlume che vacilla». La felicità nella letteratura italiana dal Quattro al Novecento, ed. V. Caputo, Franco Angeli, Milano, 2016, pp. 65-82.

VITSE, Marc, Eléments pour une théorie du théâtre espagnol du XVIIe siècle, Presses Universitaires du Mirail, Toulouse, 1990. 